\title{
Acoustic Metamaterials: A Potential for Cabin Noise Control in Automobiles and Armored Vehicles
}

\author{
Linus Yinn Leng Ang ${ }^{*, \dagger, \dagger}$, Yong Khiang $\mathrm{Koh}^{\dagger, \S}$ \\ and Heow Pueh Lee* \\ *Department of Mechanical Engineering \\ National University of Singapore, 9 Engineering Drive 1 \\ Singapore 117575, Singapore \\ $\dagger$ Kinetics Design and Manufacturing \\ Singapore Technologies Kinetics Ltd, 249 Jalan Boon Lay \\ Singapore 619523, Singapore \\ ¥e0010784@u.nus.edu; anglinus@stengg.com \\ §kohyk@stengg.com \\ Impeleehp@nus.edu.sg \\ Received 26 February 2016 \\ Revised 26 June 2016 \\ Accepted 1 July 2016 \\ Published 25 August 2016
}

\begin{abstract}
The aim of this paper is to provide an overview of the existing industrial practices used for cabin noise control in various industries such as automotive, marine, aerospace, and defense. However, emphasis is placed on automobiles and armored vehicles. Generally, automobile cabins usually constitute of thin structural panels, where the fundamental frequency typically falls below $200 \mathrm{~Hz}$. If a specific structural mode couples with a specific acoustic mode of the cabin, booming noise occurs. As such, discomfort may be felt by the occupants. Fundamentally, vibroacoustics problems may be minimized if the acoustic modes and the structural modes are decoupled, which is achieved usually by structural modifications or acoustical treatments. However, if excessively performed, the weight limitation of an automobile design will be exceeded; not to mention the adverse effect of increased weight on several factors such as fuel efficiency, mileage life of tires and acceleration of the vehicle. Moreover, current solutions have several drawbacks in low frequency noise control. In light of this, it is of great interest to explore the feasibility of acoustic metamaterials as an alternative with hope to improve cabin noise.
\end{abstract}

Keywords: Cabin noise; interior noise; noise control; vibroacoustics; review; automobile; armored vehicle; acoustic metamaterials; defense industry; automotive industry.

\section{Introduction}

In the automobile industry, both mechanical and electrical technologies have evolved dramatically since nineteenth century. Continuous and rapid cutting-edge

${ }^{\ddagger}$ Corresponding author.

This is an Open Access article published by World Scientific Publishing Company. It is distributed under the terms of the Creative Commons Attribution 4.0 (CC-BY) License. Further distribution of this work is permitted, provided the original work is properly cited. 
developments allow manufacturers to gain technological advantage and stand out from their competitors. The rate of these developments has, however, decreased and more or less arrived at a plateau lately. Existing technology and specifications are now simply a baseline in the viewpoint of most consumers. As such, greater demands have grown among other factors for purchase consideration between manufacturers. One of such factors includes passenger comfort [Shende, 2014]. This aspect is related to the consideration of human factors engineering and the study of noise and vibration within automobile cabins. Moreover, the continuous work of enhancing current automobile technologies drives the need for better noise solutions in automobile cabins for minimal weight penalty [European Automotive Research Partners Association, 2013].

Apart from the commercial consideration, the defense industry also emphasizes the need in improving passenger or occupant (referred as crew in the defense industry) comfort in the design phase of armored vehicles. This has been widely discussed as the amplitudes of cabin noise in armored vehicles - especially tracked vehicles - are way higher than commercial vehicles. In fact, safety design requirement is defined with reference to defense standards to impose a limit $(<85 \mathrm{dBA})$ for maximum allowable cabin noise under a continuous exposure of eight hours [Department of Defense, 2015]. The reason is to not adversely affect the operational readiness of the crew during a mission or any day-to-day operation. As such, crew comfort has become a marketing asset for a defense manufacturer to stand on a higher ground than its competitors.

The most common and easiest approach in reducing noise exposure by the crew is to provide them with Combat Vehicle Crewman's (CVC) helmet. These CVC helmets generally come with an in-built active noise control (ANC) module for enhanced noise reduction. In some cases, they are also advised to equip themselves with earplugs for enhanced noise attenuation if necessary. These solutions, however, are more effective in the higher frequency range $(>200 \mathrm{~Hz})$. Therefore, the crew may still be exposed to excessive cabin noise especially when a phenomenon known as booming noise is present.

In general, automobile cabins constitute of thin structural panels such as doors, windscreen, and windows. As such, the fundamental frequency of these structural members usually falls below $200 \mathrm{~Hz}$ although the cabin shape of each automobile may be unique. Cabin noise is highly dependent on the behavior between acoustic modes of the cavity and structural modes of the encompassing panels. If a specific structural mode couples with a specific acoustic mode, cabin noise is likely to amplify significantly as a result of vibroacoustics; earlier referred to as booming noise. This phenomenon is usually perceived by auditory system of humans as a loud low pitch monotone. Depending on the sensitivity of an individual to a specific booming frequency, discomfort such as nausea or giddiness may be experienced, which is evidently undesirable.

Fundamentally, vibroacoustics problems may be minimized if the acoustic modes and the structural modes are decoupled, which is achieved usually by structural 
modifications or acoustical treatments. This is seemingly easy as such studies can be performed numerically during the design phase. However, it is possible that excessive treatments may be required on specific panels due to closely-spaced acoustic modes. Inevitably, the weight limitation of an automobile will be exceeded; not to mention the adverse effect of increased weight on several factors such as fuel efficiency, mileage life of tires/track pads and acceleration of the vehicle [Vige, 2010]. In light of these, it is of great interest to explore the feasibility of an emerging research - acoustic metamaterials - with hope to improve cabin noise of automobiles and armored vehicles.

This paper aims to provide an overview of the existing industrial practices used for cabin noise control in various industries namely automotive, aerospace, and defense. Several review papers can be found in the literature pertaining to cabin noise control in automobiles but only a few highlighted the drawbacks of existing methodologies. Moreover, none highlighted the potential of applying acoustic metamaterials in the cabins of automobiles and armored vehicles [Elliott, 2008; Genuit, 2004; Hansen, 2005; Lalor and Priebsch, 2007; Rao, 2003; Sestieri and Carcaterra, 2013]. In Sec. 2, the current industrial practices pertaining to cabin noise control are discussed. In Sec. 3, the potential of acoustic metamaterials is highlighted. In Sec. 4 , a conclusion is made based on the earlier sections.

\section{Current Practices in the Industry}

Extensive studies have been conducted over the span of past decades due to the rising demand for enhanced passenger comfort in various industries namely automotive, aerospace, and defense. These studies are categorized accordingly based on the nature of their approach and discussed in the following order:

(1) Psychoacoustics

(2) Acoustical Treatments

(a) Helmholtz Resonator

(b) Sound Absorption Material

(c) Viscoelastic Damping Treatment

(d) Dynamic Vibration Absorber

(3) Active Controls

(a) Active Noise Control

(b) Active Structural Acoustic Control

(4) Numerical Methods

(a) Finite Element Method and Boundary Element Method

(b) Statistical Energy Analysis

(5) Dimensional Analysis 


\subsection{Psychoacoustics}

Conventionally, cabin noise is measured from a specific field point using microphones. Through post-processing, this measurement is then matched against safety regulation; typically A-weighted. Conversely, it is believed that such approach does not account for the subjective behavior of an individual to the perception of noise. Thus, psychoacoustics aims to consider this area by studying noise perception of the humans' auditory system. This is usually practiced in the automotive industry to shape cabin noise signature and is becoming more popular recently especially for luxury vehicles. Analyses are presented in terms of sound quality evaluation (SQE), which is expressed in terms of loudness, sharpness, roughness, fluctuation strength, speech intelligibility, and/or tonality. In the automotive industry, different methods have been proposed to improve the perception of cabin noise.

A potential approach is to consider the effect of masking by balancing interior noise components such as engine noise, booming noise, road noise, and wind noise, by the presence of another sound [Hoshino, 2000]. The focus is placed on only wind and road noise as they are claimed to be dominant cabin noise contributors at high travelling speed $(>100 \mathrm{~km} / \mathrm{h})$. However, the travelling speed of automobiles is usually much slower in an urban area with well-developed infrastructure due to regular intervals of traffic light. As such, booming and engine noise are likely to be the dominant cabin noise contributors for a huge percentage of the commute time. For armored vehicles, travelling at such high speed is even more unlikely due to their massive tonnage. Some investigated the combined effect of vibration and cabin noise as a result of chassis components [Genuit, 2004; Radic et al., 2011] and road profile [Nopiah et al., 2014, 2015].

Some works specifically targeted the sound evaluation of booming noise by proposing booming index [Hatano and Hashimoto, 2000; Shin et al., 2009]. This index is used for objective evaluation of the booming sound quality within an automobile cabin. However, correlation may sometimes be poor between the index and field measurements depending on the studied frequency range. In light of this, numerous attempts can be found to improve this approach by applying artificial neural network [Lee and Lee, 2009; Lee et al., 2006]. Concurrently, the artificial neural network is improved on by proposing to combine different methods [Paulraj et al., 2010; Wang et al., 2007; Yildirim and Eski, 2008]. Typically, psychoacoustics studies require a jury or human subject evaluation, which is time consuming since it involves surveying a specific sample audience size and compiling the feedback may be tedious. In light of this, new models and methodologies are proposed to omit the process [Allman-Ward et al., 2004; Nor et al., 2008]. Apart from automobiles, Hu et al. [2014] and Liu et al. [2013] performed similar studies on train cabins.

These works clearly showed that psychoacoustics may be a good approach for sound quality evaluation of cabins. However, such approach is only feasible when the cabin noise is not excessively high such that safety exposure limit is exceeded. 
This is likely the case in armored vehicles since crew noise is usually on or near the borderline of safety exposure limit. As such, higher priority should always be placed on crew noise reduction rather than perception studies and/or noise signature shaping.

\subsection{Acoustical treatments}

\subsubsection{Helmholtz resonator}

More than two decades ago, Fahy and Schofield [1980] highlighted several disagreements between literatures pertaining to the coupling effect of Helmholtz resonator (HR) in an enclosure. In light of this, they attempted to resolve the disagreements with an investigation to understand the influence of a HR on the acoustic mode of a rectangular enclosure. To their surprise, an interesting phenomenon is observed when the natural frequency of the HR is tuned to the frequency of an acoustic mode. Two new coupled modes are generated on each side of the tuned frequency. The frequency separation between each new coupled mode and the tuned frequency is governed by a coupling parameter and the position of the resonator with relative to the pressure anti-node of the acoustic mode. The coupling parameter widens the separation when the volume ratio of cavity-to-resonator increases. It is emphasized that the tuning of a HR should be carefully performed with strong understanding of the environment of its intended application. For instance, if only an exciting frequency exists, tuning the HR exactly to that particular frequency will be beneficial. However, if there exist more than one exciting frequencies (varying excitation), careful tuning will be necessary to achieve acceptable noise reduction. Subsequently, their work has been referenced widely as a baseline for fundamental knowledge relating to the interaction between an HR and a closed cavity. Their work is later reasoned by Cummings [1992] to be infeasible for practical applications since the likelihood of having more than one acoustic mode is high. Apart from that, these acoustic modes may not be well-separated due to high modal density in certain cases. As such, an analytical model is proposed with the consideration of more HRs in a rectangular enclosure. However, he highlighted the tremendous amount of computational effort required for the proposed model. Furthermore, the consideration of a parametric study is too tedious analytically due to a vast amount of possible variables. Answering his concerns, Driesch [2002] considered to optimize several design parameters of multiple syringe-type resonators including chamber volume, internal damping and opening area numerically, which is more efficient. These optimallydesigned resonators are then applied on a tractor cabin to validate their acoustical performance. Despite her efforts, optimal acoustical performance is only achieved if the targeted acoustic mode remains at the same frequency, which is pointed out earlier by Fahy and Schofield [1980]. This clearly implies that the design of HR has to be unique for each cabin and cautions have to be taken for optimal acoustical performance. 
Franco [1991] considered the concept of HR in an automobile to reduce cabin noise in the low frequency range $(<200 \mathrm{~Hz})$ by adding a long rectangular slit on the topmost panel of the boot compartment. As such, a connection is established between the respective air volumes - passenger and boot compartments. However, the cabin noise is reduced by only a negligible amount of about $1 \mathrm{~dB}$ in the studied frequency range. The work is limited to the tuned frequency of the HR, which is dependent on the volume of the boot compartment and the opening area of the slit. Optimization studies are conducted to account for these parameters [Kamci, 2009; Kopuz and Lalor, 1995]. Generally, in automobile design, the volume of the boot compartment is usually optimized for maximum room space. This, in turn, will become a design issue if a smaller boot compartment is required to tune its natural frequency upwards to match a targeted acoustic mode of the passenger compartment. Or in another case, a bigger boot compartment to match a low frequency acoustic mode. Either ways, the approach may not be that effective since the amount of cabin noise reduction may still be insignificant. Furthermore, debates will arise whether to compromise storage space for improved cabin noise. Nonetheless, one can also consider enlarging the slit to have an opposite effect on the natural frequency of the HR. Despite the fact, there is always a limit to the largest allowable slit area since the effect of a HR may diminish when the silt area becomes too large. In most armored vehicles, a boot compartment may even be unavailable since the crew are expected to carry their own belongings. On the contrary, one may still suggest the possibility of adding empty compartments within the crew cabin for HR effect. Hence, the overall crew space will be affected unnecessarily, which leads to difficulty in the ingress/egress movement of the crew. On the other hand, if slits are made on the cabin walls, crew survivability may be compromised.

From here, it is evident that multiple HRs may indeed be more effective for noise reduction in a large enclosure as mentioned by Cummings [1992]. As ideal as it may sound, it is worth noting that the addition of more HRs into an automobile cabin may raise design issues pertaining to space and maybe even weight constraints. Both are certainly undesirable. In light of this, Doria [1995] put forward an analytical solution by connecting two expansion chambers in series in the form of a linear duct assembly. The opening of the resonator is then exposed to the enclosure wall. The key idea is to achieve equivalence to one resonator yet possessing the characteristics of two. Again, the volume of each expansion chamber has to be carefully tuned to achieve optimum noise reduction in the rectangular enclosure. On top of that, the positioning of the resonator on the enclosure wall is also important as a poorly-placed resonator may adversely influence the acoustic characteristics of the enclosure. The challenge of tuning the volume of each expansion chamber accurately is highlighted; particularly in realizing them experimentally. This is due to geometric deviation as a result of inevitable machining tolerance. Nonetheless, such is no longer a concern with the current manufacturing technology. In an automobile, limited positions may be available for proper placement due to the inherent positions 
of other structural features or electrical components. This issue worsens in armored vehicles as even more vetronics systems are present. Evidently, the consideration of practical installation by earlier works seems to be lacking.

Recently, Chintapalli and Padmanabhan [2014] proposed the use of multiple smaller resonators; each tuned similarly to the targeted acoustic mode of a rectangular enclosure. They showed significant noise reduction albeit it becomes negligible beyond three resonators. Hence, the acoustical performance of their resonators may deteriorate further if extended to larger enclosures. Separately, Yu et al. [2009] proposed T-shaped resonators that aims to remain compact in geometry yet not compromising acoustical performance. They started off by establishing an analytical model [Li et al., 2007; Li and Cheng, 2007] for multiple resonators within an enclosure based on an earlier work [Fahy and Schofield, 1980]. Concurrently, their analytical model is validated experimentally by several designs of T-shaped resonators. Likewise, they showed the importance of proper positioning of the resonators within an enclosure for optimum noise control. This is consistent with the findings by Doria [1995]. Moreover, they also highlighted the influence of internal resistance on the acoustical performance of the resonators. These issues are discussed in detail subsequently [Yu and Cheng, 2009; Yu et al., 2008]. To demonstrate the effectiveness and feasibility of their proposed resonators, further work [Li et al., 2010] is carried out by incorporating the resonators into a double-leaf panel to attain improved narrowband noise attenuation at targeted low frequencies $(<400 \mathrm{~Hz})$ depending on the tuning of the resonators. Although the spatial requirement due to its small cross-sectional area is attractive, the physical length of the resonator increases as the targeted frequency gets lower (up to about $40 \mathrm{~cm}$ for noise attenuation at $200 \mathrm{~Hz}$ ). Similarly, in the aerospace industry, the concept of HRs is also studied by different research groups [Esteve and Johnson, 2002, 2005; Howard et al., 2005; Lane et al., 2005; Park and Sohn, 2010; Park and Sohn, 2009; Park, 2013a, 2013b; Sohn and Park, 2011].

In some works [Fenech et al., 2006; Liu and Herrin, 2010; Wang et al., 2010; Yang et al., 2015], the use of micro-perforated panels (MPP) are proposed, which are based on the fundamentals of HR. In this case, each perforation effectively represents a resonator with the backing air cavity representing the expansion chamber of a conventional resonator [Arenas and Crocker, 2010]. The potential of MPP is recently discussed in detail by Maa [2015]. In this paper, the key design parameter is the influence of open-area ratio on the acoustical performance of a MPP, where an optimum sound absorption is achieved at specified open-area ratio. This is aligned with the findings of other recent works [Bravo et al., 2012, 2013; Zhao et al., 2015]. However, the difficulty in realizing MPP with much smaller orifices $(<0.3 \mathrm{~mm}$ in diameter) is a concern due to limitations of current manufacturing techniques; including additive manufacturing methods. It should be noted that this requirement may only be necessary if one is able to compromise for a narrower bandwidth of sound absorption with larger orifices. On the other hand, Yu et al. [2014] showed otherwise by considering a multi-layered MPP configuration arranged in a staggered formation. Despite the success of MPP in duct applications [Cox and D'Antonio, 
2009], the depth of the backing air cavity has to be sufficiently generous for effective low frequency attenuation. Again, cabin space is affected. The inevitability of a backing air cavity could, however, be potentially minimized or omitted based on the recent work by Toyoda et al. [2015] albeit extensive further studies are necessary for such realization in panels. From these works, MPP seems to be an improved concept of HR albeit the minute orifices may likely trap dust particles. As such, one should also account for the ease in cleaning the panels especially if they are installed in the cabin interior or at tight corners.

It is evident that the application of resonators into automobiles and armored vehicles is likely to raise inevitable issues pertaining to (i) spatial requirement, (ii) the increase in vehicular weight with increasing number of resonators, (iii) the effort required for design optimization, and particularly for MPP, (iv) the ease of cleaning. The lack of literature on actual application for cabin noise control in automobiles and armored vehicles is evident that such approach is not practical enough to be accepted by manufacturers. Moreover, the total number of resonators increases with cabin size as well. Aside from these apparent concerns, extensive effort will most probably be necessary to incorporate multiple resonators in a cabin while maintaining interior aesthetics to the potential buyers.

\subsubsection{Sound absorption material}

Sound absorption material - a common and traditional passive noise solution found in the industry — is usually available in sheets made of a porous medium such as fiberglass or polyurethane foam. As sound waves propagate within the porous network, part of the sound energy is dissipated due to viscous and thermal losses [Arenas and Crocker, 2010; Cox and D'Antonio, 2009]. As such, the transmitted or reflected sound waves are reduced in sound energy. Depending on the area of application, several parameters, such as porosity, density and, thickness, can be varied to achieve intended acoustical performance. Being easily implementable and cheap makes them attractive as a preliminary measure for cabin noise control. As the discussion of a detailed theoretical background is beyond the scope of this paper, interested readers can refer to specialized literature for further perusal [Arenas and Crocker, 2010; Cox and D'Antonio, 2009; Egab et al., 2014].

There are numerous analytical works that demonstrated the influence of dissipative material on the walls of an enclosure [Bruneau et al., 1985; Du et al., 2011; Lanoye et al., 2008; Martin and Bodrero, 1997; Pan et al., 1999; Suzuki et al., 1989]. They showed that the interior noise of an enclosure can be reduced especially if the placement of sound absorption materials is optimized. Later, Sarigul and Kiral [1999] extended these analytical concepts to a two-dimensional model for cabin noise control in a truck cabin. Sound absorption materials are considered only on the floor and rear wall for practicality as the sides are usually the doors. Although considerable noise reduction is presented at $95 \mathrm{~Hz}$, they provided no concise details on the acoustical characteristics and thickness of the modeled material. As such, 
the results may be questionable since it is known that a reasonably thick layer of sound absorption material is required for noise attenuation at such low frequency to be at least noticeable. More realistic results are demonstrated in an excavator cabin [Willemsen et al., 2009] and a van [Cherng et al., 2002]. Apart from automobile applications, this solution is also considered in aircraft fuselage [Boily and Charron, 1999] and train cabin [Hu et al., 2014]. Exclusively, active sound absorption material, known as smart foam, is also considered in aircraft fuselage [Gentry et al., 1997; Guigou and Fuller, 1999; Kim et al., 1999].

The necessity of having a thick layer of sound absorption material is proven otherwise by a research group that proposed a so-called "double porous material" [Atalla et al., 2001]. The material is constructed simply by introducing periodic arrays of cylindrical cut-outs similar to that of MPP albeit in the macroscale for this case. Despite the difference in the shape of the cut-outs (square) considered in their numerical model, they showed enhanced low frequency absorption coefficient of up to 0.9 - specifically between 200 and $300 \mathrm{~Hz}$ - with a thickness of $11.5 \mathrm{~cm}$. This finding is noteworthy due to the fact that a higher sound absorption coefficient is achieved in the lower frequency range as compared to the higher frequency range. This differs from the sound absorption profile of conventional sound absorption materials. In light of this, they performed a parameter study to understand the influence of cut-out size and its distribution. It is concluded that a smaller size is preferred for low frequency noise control and sound absorption performance is observed to deteriorate when periodicity is removed. Several subsequent works in all aspects - numerical, experimental and analytical — are performed by the same research group and is later compiled as a review paper, which serves as a design guideline for interested researchers [Sgard et al., 2005]. The success of sound absorption material in mid and high frequency noise attenuation is well-known albeit documentation of the case studies is usually presented in company websites, brochures, and catalogs. As such, limited reported literature can be found pertaining to their applications in the industry.

Recently, the inclusion of HRs in porous material is proposed by arranging them similarly in a periodic manner [Doutres et al., 2015]. Expectedly, excellent sound attenuation is achieved but limited to the tuned resonance frequency of the resonators. Owing to the acoustical performance of the porous material in the higher frequency range, the obvious advantage of such design is to also have an enhanced yet limited bandwidth of low frequency absorption. For example, they achieved an insertion loss of up to $8 \mathrm{~dB}$ at $145 \mathrm{~Hz}$ in one of their configurations. From their findings, it is evident that the proposed design may be more suitable in applications where the control of tonal noise is of concern. On the side note, the construction of this design may pose numerous challenges such as the alignment (angle) of each resonator with respect to the incident wave and maintaining a uniform depth of placement for each resonator within the porous material.

Apart from this recent work, hardly any reported studies can be found on the potential of sound absorption material for low frequency $(<200 \mathrm{~Hz})$ noise control 
without compromising weight gain as a result of increased thickness. Moreover, the degradation of their composition with time is another issue that affects reliability and acoustical performance in the long term basis. This is especially the case in armored vehicles as their mobility is usually in harsh environment. Frequent maintenance is definitely not favored.

\subsubsection{Viscoelastic damping treatment}

The benefits of viscoelastic damping treatments are comparable to sound absorption materials; particularly relating to cost, simplicity, reliability, and stability. However, their working principle is unlike sound absorption materials. They are typically applied to the targeted panel to reduce vibrational energy and, in turn, reduce sound radiation into the enclosure. In general, there are three main types of such damping treatments - free-layer damping (FLD), constrained-layer damping (CLD) and tuned viscoelastic damper (TVD).

FLD is applied by spray-coating onto a targeted panel. Vibrational energy is dissipated by its extension and compression along the plane of the vibrating panel. CLD consists of a layer of viscoelastic material sandwiched in between two outer constraining layers. When a panel vibrates, vibrational energy dissipation occurs due to shearing deformation as a result of the outer constraining layers. TVD acts like a mass-spring system, where a tuning mass is attached to the targeted panel by a layer of viscoelastic material. This viscoelastic material effectively acts as a spring. The natural frequency of the system is then tuned to an excitation frequency. As such, prior knowledge of the vibrational profile in the base structure is essential. Similar to the coupling effect of HR, the excitation frequency is separated into two sidebands if properly tuned.

Out of these, CLD is commonly used in the industry due to the presence of additional damping as a result of shearing deformation as compared to FLD of identical weight [Rao, 2003]. Supporting this statement, the effectiveness of CLD is evident from literature with it being considered in various transport systems such as ship [Pang et al., 2014], tractor [Cao, 2005], truck [Subramanian et al., 2004], aircraft [Boily and Charron, 1999], and helicopter [Caillet et al., 2012]. Some even attempted to introduce active control system into CLD [Poh et al., 1996]. On the other hand, TVD is less popular albeit applications can still be found in aircraft [Halvorsen and Emborg, 1989] and payload fairing [Howard et al., 2005]. This trend is possibly explained by the added advantages of dynamic vibration absorber (DVA), which is technically simpler in design. Nonetheless, it is noted that viscoelastic damping materials are sensitive to temperature changes and may, in turn, exhibit changes in inherent damping properties. As such, damping performance may no longer be optimal in a harsh environment encountered by armored vehicles; implied by the lack of application in this area. 


\subsubsection{Dynamic vibration absorber}

Generally, the underlying concept of DVA is similar to TVD in the sense that both are made up of a mass-spring system. However, they should not be confused as identical since DVA does not require damping to achieve intended functionality. This implies the preference of DVA over TVD due to very limited literature for the latter. Moreover, discrepancy between numerical and experimental results can be reduced as the need for modelling viscoelastic damping material in DVA is not required. Being a passive solution, it has similar benefits with those as discussed earlier.

The use of DVA is demonstrated by Huang and Fuller [1997, 1998] on the walls of a cylindrical enclosure to reduce vibration magnitude. Depending on the type of excitation, optimum performance is achieved by either placing numerous lightweight absorbers in close proximity to a heavyweight absorber or distributing them in a scattered manner on the enclosure walls. On the contrary, a later work by the same group realized further noise reduction by mistuning the DVAs [Fuller et al., 2000]. Consistently, they indicated the influence of positioning the DVAs on optimum noise reduction, which is extended by Huang and Chen [2000] albeit the consideration of heavyweight absorbers is not included. However, their findings are based on numerical and analytical predictions without any experimental validation. In another work, Esteve and Johnson [2002, 2005] proposed the combination of DVAs and HRs to enhance noise reduction but in this case, their predictions are validated experimentally though in a cylindrical enclosure instead of an actual aircraft. Sacarcelik [2004] performed a more comprehensive study with validation of his predictions on an actual payload fairing.

All these works showed the effectiveness of DVA in reducing cabin noise by controlling panel vibration. However, similar to HR, they are only effective when the excitation is constantly maintained at the same frequency. Any deviation from the tuned frequency will cause a reduction in the overall performance. Moreover, depending on the size of the enclosure, multiple DVAs are required and strategically placed to achieve effective cabin noise reduction. With a smaller cabin size in automobiles and armored vehicles as compared to aircrafts, one may relate to proportionality and suggest lesser required DVAs. This may not be the case since vibration input into these vehicles is varying. As such, more DVAs may be required instead to achieve broadband control. However, to place them optimally, identical issues will arise as highlighted earlier for the case of using HR.

\subsection{Active controls}

The benefits of conventional passive treatments, such as cost and simplicity of installation, are undebatable. However, the acoustical performance of passive controls may be limited to high frequency with a narrow low frequency bandwidth. For an excitation that varies in frequency, passive control is still possible as highlighted earlier 
but proper considerations must be emphasized during the design phase. Active control is an improvement to passive control for its ability to sense vibration or sound pressure level of a specific field point and generate an output that is of opposing magnitude or phase to counter them. Cabin noise can either be controlled directly by an array of secondary sources or by reducing structural vibration with some form of active actuators. Generally, the former is commonly known as active noise control (ANC) and the latter as active structural acoustic control (ASAC).

\subsubsection{Active noise control}

Typically, microphones are mounted in the cabin to measure interior noise. The measured noise signal is then fed into a controller, where an anti-phase signal is then generated to several loudspeakers for transmission. The loudspeakers are positioned strategically in the cabin; usually in close proximity to the control region. In reality, perfect correction is impossible due to the possibility of phase lag or other contributing factors. As such, the microphones concurrently measure the corrected cabin noise and repeat the whole process. This process allows ANC to be achieved. In control theory, the microphones and loudspeakers are referred to as error sensors and secondary sources respectively. The number of error sensors and secondary sources is dependent on the cabin size. This implies that the number of error sensors and secondary sources is increased for the case of a large cabin. Conversely, increasing the number of error sensors and secondary sources in a fixed cabin size may yield better noise control. The discussion of a detailed theoretical background is beyond the scope of this paper. Interested readers can refer to the literature for further perusal [Muller and Moser, 2013].

There are two kinds of controller — namely feedback and feedforward [Sutton et al., 1994; Tichy, 1991]. The former is a very simple control system, where it involves secondary sources and error sensors as described above. Despite its simplicity, prior knowledge of the primary source is mandatory and the secondary sources must be placed in close proximity to the error sensors to prevent phase lag, which eventually drives the system into instability. Hence, robustness becomes a problem. Feedforward control is more complex, where an additional reference sensor is required to measure the primary source and subject the signal to an algorithmic filtering. The filtered signal is then transmitted through the secondary sources. Concurrently, the error sensors will pick up the combined sound field of the primary and secondary sources and adjust the filtering accordingly. In a way, this may be known as adaptive control, which is desirable for frequency dependent primary sources. The advantage of such approach is that cancellation only focuses on the reference signal rather than the global noise signature, which may include other components such as speech or music. However, for this to be ideal, the correlation of the reference 
signal with the primary source must be near unity. Understandably, this is a huge challenge.

In the aerospace industry, countless research efforts are placed on improving the interior noise comfort of a fuselage, specifically in the passenger compartment, since 1990s. On a scaled-down basis, the feasibility of ANC is considered in a cylindrical enclosure using a feedback control system [Silcox et al., 1989]. Conversely, another research group demonstrated likewise on a full-scale twin turbo-prop aircraft [Dorling et al., 1989]. Targeting the tonal excitation of the propeller $(88 \mathrm{~Hz})$, a maximum reduction of up to $11 \mathrm{~dB}$ is achieved. Concurrently, another work demonstrated likewise on a different aircraft but with lesser secondary sources [Elliott et al., 1989]. They claimed that the sum of squared measured sound pressure can be minimized in this way. Similarly, they considered a tonal excitation source $(88 \mathrm{~Hz})$ and achieved a maximum reduction of up to $14 \mathrm{~dB}$. Evidently, these preliminary works showed the potential of ANC for low frequency cabin noise control. With the potential of ANC shown experimentally, Bullmore et al. [1990] put forward an analytical model to validate the results of earlier work [Elliott et al., 1989]. Good agreement is observed in their validation. To their surprise, they noticed a high level of local reduction around the error sensors. A later paper compiled all these works and investigated their observations [Elliott et al., 1990]. Later, the dependency of global cabin noise reduction on the number of secondary sources is investigated in two different aircrafts [Martin et al., 1994]. Numerically, an incremental gain in the global reduction is computed, which agrees with experimental results up to fifteen secondary sources. The computed reduction remains constant beyond fifteen secondary sources. Although modelling assumptions may be the cause, no clear explanations are provided.

To achieve global attenuation within a cabin, an extensive amount of sensors is necessary. This is inevitable though undesirable since the bulkiness of the control system naturally increases as well; not to mention the increase in complexity as well. Weight is of high design priority in the aerospace industry since it affects fuel efficiency and payload capacity. Taking into consideration of the findings by Bullmore et al. [1990], a possible solution is to adopt a local control by placing error sensors near the head of a flight crew or passenger. This solution is somewhat reasonable since the motion of a flight crew or passenger usually remains around the same spatially. However, a new problem arises as the control region generated by the error sensors is usually insignificant that no noticeable improvement can be felt. In light of this, virtual sensors are proposed not only to solve this limitation but also to broaden the control bandwidth [Kestell, 2000]. Additionally, they are designed to track the motion of one's head and translate the control region accordingly. This is done by predicting the forward wave. However, the work is limited to a onedimensional field and is highlighted of the potential challenges in extending it into a three-dimensional field. Lane et al. [2000] considered otherwise by attempting to improve the performance of the controller for better cabin noise control with more 
complex algorithm. Apart from commercial aircrafts, similar studies are done on a military cargo aircraft using a feedforward control system [Gerner et al., 2003; Kochan et al., 2010, 2011].

In the automotive industry, ANC is also reported in numerous works. The feedforward control system is demonstrated on the front seats of a commercial car by Sutton et al. [1994]. Despite the effectiveness of the control system below $200 \mathrm{~Hz}$, they highlighted that it is not sufficiently robust and reliable as instability occurs even with a slightest delay of milliseconds. Lee et al. [2014] and Oh et al. [2002] performed similar studies by considering, instead, improved algorithms. Evidently, earlier works validated the potential of ANC but proper consideration of distributing the secondary sources and error sensors in the cabin has yet to be considered. Stanef et al. [2004] demonstrated an optimization study in a mining vehicle. They found that significant cabin noise reduction can be achieved if the secondary source is placed in close proximity to the primary source. Their findings resonate with the earlier observations by Elliott et al. [1989]. If this distance exceeds half the wavelength of interest, negligible reduction will be noticed due to high modal overlapping. Moreover, this issue cannot be solved by simply introducing more secondary sources or error sensors. As such, they concluded that global control is not feasible in a three-dimensional field especially for scenarios where modal density is high in the low frequency range. This clearly identifies the limitation of ANC for cabin noise control in spite of the decent acoustical performance in the lower frequency range.

Till date, the idea of ANC is not as well-received as expected in the automotive industry due to high cost of implementation and complexity of cabin sound field [Rao, 2003]. This especially repels consumers away in the purchase consideration of low- and mid-end cars, where extra cost is added into the total purchasing price. A brilliant attempt to reduce the installation cost of feedback control system is proposed by incorporating it into the in-car entertainment system [Cheer and Elliott, 2013]. They developed three different kinds of feedback control systems of varying complexity and validated their performance in a commercial car. The idea is certainly innovative by exploiting existing built-in audio systems, which is almost a necessity in any typical commercial car for musical pleasure. However, no details are provided pertaining to the estimated cost of each controller.

The drawbacks are clearly identified by these works with more evidences from review papers [Elliott, 2008; Hansen, 2005] and magazine articles [Hughes, 2009; Noise \& Vibration Worldwide, 2002] highlighting numerous hurdles to overcome before ANC is welcomed with open arms by the automotive industry. Some of these reasons include complex hardware configurations, high installation/implementation cost, non-user-friendly software, complex algorithms, unique controller for different cabins, need for external power source, and non-ruggedized control systems. It is highly recommended that one should consider ANC only if attempts in implementing passive solutions are exhausted or impractical since the return of investment 
for ANC is highly uncertain. Noting the last point from the list of challenges, the application of ANC in armored vehicles is even more challenging if the control system is not sufficiently ruggedized to withstand harsh environmental conditions experienced commonly during mobility.

\subsubsection{Active structural acoustic control}

ASAC is more advantageous as compared to ANC due to its characteristic in controlling both cabin noise and structural vibration simultaneously. ANC does not influence structural vibration and hence if cabin noise is dominated by sound radiation of the panels, the acoustical performance of the control system may not be that effective. Generally, the working principle is similar to ANC. Accelerometers are attached onto the targeted panel to measure the vibration amplitude due to a sound source. The measured signal is then transmitted to a controller to generate an anti-phase signal, which becomes the vibration output of a mechanical actuator attached to the same panel. As such, a secondary vibration input onto the panel to reduce its vibrating amplitude and minimize sound radiation into the cabin. Accelerometers are placed on the panel to capture and feedback the corrected vibration signal to the controller. This approach is more common in the aerospace industry with applications extending to space shuttles; whereas literature is very limited in automotive applications.

Fuller and Jones [1987] first demonstrated the potential of ASAC on a cylindrical enclosure experimentally. Their control system involves a mini-shaker and several accelerometers mounted onto a targeted panel. An external sound source simulates tonal engine noise into the enclosure, which in turn excites the encompassing panels into vibration. Accelerometers pick up and transmit the vibration signal to a controller, which eventually generates an anti-phase signal to the mini-shaker. As a result, sound radiation from the targeted panel is minimized. To their surprise, global interior noise control is observed albeit only one actuator is used. They then extended the work to a full-scale aircraft for validation [Simpson et al., 1991]. Concurrently, another research group demonstrated analytically and experimentally at a higher level of detail in a rectangular enclosure; including optimizing the position of the actuator [Pan et al., 1990; Pan and Hansen, 1991; Pan, 1991]. They found that the optimum performance can be achieved from more than one position.

Earlier works proved the feasibility of ASAC for cabin noise control but mechanical shakers are typically bulky and heavy. Hence, piezoelectric patches are proposed in place of mechanical shakers to achieve the same functionality [Fuller et al., 1992; MacMartin, 1996; Mathur and Troi, 1993]. The performance of piezoelectric patches are proved to be comparable to mechanical shakers if properly designed, which brings the technology of ASAC to a whole new level with reduced bulkiness [Sun et al., 1996]. Apart from being compact, piezoelectric patches require lesser external power to be driven as compared to mechanical shakers [Balachandran et al., 1996]. Expectedly, piezoelectric patches are in the limelight and mechanical shakers are 
replaced gradually in ASAC systems with time. This is evident from later literature found on aircrafts [Grewal et al., 2000; Kincaid et al., 1997; Palumbo et al., 2000], payload fairing [Niezrecki and Cudney, 2001], and commercial cars [Sas and Dehandschutter, 2009; Song et al., 2003].

At this juncture, it is understood that cabin noise can either be achieved by ANC or ASAC. However, there is no comparative work to distinguish them clearly with regards to their respective benefits. In light of this, Sas and Dehandschutter [2009] attempted to answer this with a demonstration on a commercial car. Based on their numerical and experimental results, they found that ANC is able to achieve higher cabin noise reduction but limited to the vicinity of the error and reference microphones. This is in agreement with earlier observations by Bullmore et al. [1990]. Conversely, ASAC provides a more consistent global cabin noise reduction. From here, light is shed for researchers to consider each approach depending on their objective. Misol et al. [2012] considered differently another comparative study to understand and distinguish the acoustical performance of feedback and feedforward controllers by implementing them on a windshield of a commercial car experimentally. Although comparable results are achieved by both types of controllers, the effectiveness reduces when the vibrating pattern of the windshield becomes more complex. This is somewhat similar to one of the drawbacks of ANC with reduced performance in complex cabin sound field.

Among the various active solutions, ASAC still stands out as it is able to kill two birds with one stone by reducing both structural vibration and cabin noise simultaneously. However, similar to other active control systems, they do have their shortcomings. Also, ASAC may be detrimental to the structure as secondary vibration input may induce additional fatigue damage [Hirsch et al., 1998]. This shortens the life of the structure, which eventually leads to increased maintenance cost. In the perspective of armored vehicles, the consideration of ASAC may not be wellaccepted than ANC since increased fatigue damage into the hull may indirectly affect crew survivability.

\subsection{Numerical methods}

First and foremost, it should be noted that the emphasis of this paper is to provide an overview of the current practices used in the industry for the study of cabin noise. As such, a detailed account of past analytical studies is of irrelevance to this paper. Moreover, the industry relies dominantly on the usage of computational software rather than analytically obtaining results from mathematical formulations. A tremendous amount of time will be consumed in reality since cabins are usually irregularly shaped with complex boundary conditions [Atalla and Bernhard, 1994]. Conversely, one should not forget the importance of analytical frameworks developed by early researchers since the success of computational software is built based on them. Extensive literature can be referred to for interested readers to understand the analytical aspect of numerical methods [Dhandole and Modak, 2007; Dowell 
et al., 1977; Everstine, 1997; Kipp and Bernhard, 1987; Kopuz and Lalor, 1995; Missaoui and Cheng, 1997, 1999; Nefske et al., 1982; Pan et al., 1999; Petyt and Koopmann, 1977; Richards and Jha, 1979; Sestieri et al., 1984; Shuku and Ishihara, 1973; Succi, 1987; Wu et al., 2000].

Generally, there are three main numerical methods that are adopted widely by the industry for cabin noise prediction. They are namely finite element method (FEM), boundary element method (BEM), and statistical energy analysis (SEA). Geometrical acoustics techniques, such as ray tracing or beam tracing, are typically used for architectural (or room) acoustics. Although a room is essentially an enclosure, these techniques are only accurate if the targeted wavelength is way smaller as compared to the dimensions of the enclosure [Lalor and Priebsch, 2007]. Thus, they are unsuitable for typical cabins, which are much smaller in size than a room or hall. As such, it is beyond the relevancy of this paper to include geometrical acoustics techniques.

\subsubsection{Finite element method and boundary element method}

From literature, it is evident that analytical works have declined since the late 1990s as later reported works start to make use of computational software; developed based on the analytical efforts of early researchers. Kim and Lee [1998] attempted to develop a FEM software to predict cabin noise of a scaled commercial car. They validated their software experimentally by considering several boundary conditions and showed the feasibility of their software for such study. However, Cherng et al. [2002] argued otherwise with the importance of using formulations to predict acoustic modes of a given cabin analytically using classical formulations. Despite their argument, it is evident in their work that analytical accuracy drops with increasingly complex cabin shape; prominently at higher-order modes. In support of their standpoint, they stated that accurate predictions are not really essential during the design phase of an automobile, where approximated results are reasoned to be sufficient. In argument, their views may no longer be valid when the coupling effect of vibroacoustics is considered. Moreover, a physical full-scale prototype is usually unavailable for validation in industrial reality. As such, accuracy of the model is mainly governed by the working experience of the analysis engineer to define appropriate boundary conditions and assumptions. Hence, if the deviation of the model is too large, drastic design changes may be necessary after the validation stage, which is most probably too late. Even if it is still possible in the prototype stage, higher cost will be incurred for design changes.

As a rule-of-thumb, the recommended number of elements per wavelength is ten for an accuracy of about 10\% [Nefske et al., 1982]. Later, this is debated in detail by Marburg [2002] with six being reasonably acceptable. This greatly reduces computational time for larger models. Since the frequency range of interest is generally below $200 \mathrm{~Hz}$ for cabin noise studies, coarse mesh is more than sufficient for accurate prediction of acoustic modes. However, to consider the coupling effect of 
vibroacoustics, finer mesh is more favorable. This is usually done by combining FEM and BEM (FEM-BEM). Unlike FEM, BEM (based on Helmholtz integral equation) requires only the boundary conditions and discretization of encompassing panels to predict either interior or exterior noise at an arbitrary acoustic field point. As such, the original three-dimensional FEM problem is reduced to a two-dimensional BEM problem. In this manner, preparation and computational time are greatly reduced [Atalla and Bernhard, 1994]. BEM can be further categorized into two different types - namely direct (DBEM) and indirect (IBEM). The former considers a closed volume and predicts either the interior or exterior noise at one time while the latter is able to predict either side simultaneously and does not require a closed volume.

An example is demonstrated on a harvester cabin by Desmet et al. [2003]. They considered between coupled, semi-coupled, and uncoupled models and matched the results against field measurements to understand the accuracy of each model. They found that the consideration of either semi-coupled or coupled models is crucial in achieving accurate predictions. Their observation is further supported by the results of Liu et al. [2006] with their comprehensive study on an armored tracked vehicle. They considered a semi-coupled model as the influence of structural modes on acoustic modes is claimed to be more dominant than the other way round. The agreement of their numerical and experimental results validated their assumption. Kim et al. [2012] and Wang et al. [2010] demonstrated similarly on construction vehicles and bus respectively.

Liu et al. [2006] proposed the use of structural intensity to visualize vibrational power flow in the encompassing panels of a scaled automobile in the form of streamlines; analogous to that used in fluid dynamics for fluid flow studies. From the visualization, they proposed the placement of a damper at critical spot to channel the flow of vibrational energy towards it. Seemingly, vibration amplitude at selected panels is reduced. However, their work remains as a numerical study without any further work in the same industry. A more efficient FEM-BEM approach is proposed using acoustic transfer vectors (ATV) and modal acoustic transfer vector (MATV) [Citarella et al., 2007]. ATV allows the generation of a transfer function between a structural field point and an acoustic field point in the cabin. This is advantageous because the recalculation of sound pressure level at a specific field point in the acoustic field is no longer necessary since the transfer function is valid as long as the acoustic field remains unmodified. This transfer function is not affected by any change in excitation forces on the structure or modifications. As such, computational time is reduced. The approach based on MATV works on a different concept where the transfer function is generated based on the modal response at a structural field point and an acoustic field point in the cavity. This provides a convenient approach as modal response of the structure, which is obtained either from modal test or numerical prediction, can be imported into the model. Hence, the need to compute normal surface velocities of the structure is not required. The 
drawback of this approach is its dependency on modal response, where the transfer functions become invalid upon any structural changes.

The focus from most of these works tends towards the prediction of cabin noise or acoustic/structural modes. Minimal attention is placed on improving cabin noise subsequently with the predicted results. In light of this, researchers took a step forward mainly by structural modifications. This is preferred in the design phase since the process of remodeling is relatively easy. In contrary, the process may be iterative if the analysis engineer has insufficient working experience. Generally, it involves the addition of stiffeners to increase the vibrating frequencies of selected panels. On a downscale basis, Demirkan et al. [2010] and Park et al. [2010] demonstrated separately on an enclosure whereas others demonstrated on commercial vehicles [Olatunbosun et al., 2011; Sung et al., 1994] and tractor [Cao, 2005]. On the side note, some proposed differently by modifying the trim in commercial cars [Choi et al., 1997; Kang et al., 2000] or by optimizing the boundary conditions of stiffened panels [Chandra et al., 2015; Zheng and Wei, 2013]. All these works showed the effectiveness of structural modification in decoupling structural modes from acoustic modes. However, the added stiffener may not be always effective if its position on the targeted panel is not properly considered and optimized.

Lately, panel acoustic contribution analysis (PACA) is used as an additional tool of the FEM-BEM approach. It determines the significance of a panel's contribution to a specific acoustic field point in the cabin by categorizing the panel as positive, neutral or negative. Positive indicates an increase in sound pressure at an acoustic field point when the vibration amplitude of a panel increases. Negative indicates likewise for the sound pressure but when the vibration amplitude of a panel decreases. Neutral is rather self-explanatory, which simply indicates no influence on the sound pressure with any modification to the panel. The focus of structural modifications or acoustical treatments must be placed on the positive panels since their vibration amplitude directly increases the pressure level at an acoustic field point in the cabin. Its benefits and conveniences have been proven by recent works mainly on commercial vehicles [Guo et al., 2012; Han et al., 2012; Jahani et al., 2013; Xu et al., 2015; Yuksel et al., 2012]. The optimization of cabin shape may also be considered as proposed by Zhang and Cheng [2015].

\subsubsection{Statistical energy analysis}

Being a statistical approach, the underlying concept of SEA is dissimilar from deterministic approaches like FEM and BEM. The acoustic cavity and encompassing structures are divided into network of coupled subsystems; each having similar number of same mode types. Solutions are obtained based on the principle of energy conservation. This computation is usually faster than deterministic approaches since the number of degree of freedoms is further reduced [Vige, 2010]. As such, the use of SEA is beneficial either for acquiring quick results for an iterative design process or matching cabin noise against safety regulations for the auditory system of humans. 
One such example is demonstrated on a commercial car by Chen et al. [2011]. They showed good agreement against experimental results from $200 \mathrm{~Hz}$ to $20 \mathrm{kHz}$ and concluded that the feasibility of SEA for high frequency cabin noise analysis. Their conclusion resonates with that of Atalla and Bernhard [1994]. Marzbanrad and Beyg [2012] attempted likewise on another commercial car albeit they lacked validation of their numerical results. SEA is also applied for similar intention on ship [Pang et al., 2014] and trains [Forssen et al., 2012; Zhao et al., 2013]. Interest in improving the methodology of SEA is still ongoing; evident from recent literature [Putra et al., 2015]. On the other hand, others attempted a different approach in improving SEA by combining it with FEM to achieve higher prediction accuracy in the lower frequency range [Blanchet and Caillet, 2014; Chen et al., 2011; Lalor and Priebsch, 2007; Zhao et al., 2013]. As such, the entire audible range of hearing can be covered $(20 \mathrm{~Hz}$ to $20 \mathrm{kHz})$. At this point, one may notice the lack of literature pertaining to armored vehicles since, factually, concerns are always in the lower frequency range $(<200 \mathrm{~Hz})$.

\subsection{Dimensional analysis}

This subsection is included for the intent of coverage since some researchers have been attempting to predict cabin noise using a different approach. In the industry, physical prototypes are usually not available in the design phase and justifying for a mock-up to be fabricated solely for noise and vibration studies may sometimes be difficult due to additional design cost. With this in mind, researchers [Georgiev et al., 2006a, 2006b; Krylov et al., 2003; Krylov, 2002, 2004] proposed to apply dimensional analysis to obtain a simpler downscale model of an automobile, which possesses a different cabin shape as the original. The downscale model allows them to perform prediction of cabin noise and correlate the results back to the full-scale cabin. There are two main considerations in performing cabin noise prediction in downscale models - degree of model's complexity and accuracy of prediction. One should note that increasing the degree of model's complexity may indeed yield more accurate results but in the expense of computational cost. A balance has to be achieved between these two factors.

In their work, the downscaled model is known as quarter-scale interior cavity acoustic rig (QUASICAR). First, an analytical model is derived based on the principles of Green's function, Helmholtz equation and acoustic wave equation to predict the interior noise level of the QUASICAR. In their analytical model, they assumed that the feedback of acoustic response has no influence on the structural response of the boundary and smallest radius of the plate curvature is large in comparison with the flexural wavelengths of interest so that shell-type behavior shall not be accounted for. Subsequently, they extended to experimental investigation and found that the measured acoustic modes are of good agreement with their analytical prediction but is otherwise for the structural modes. This disagreement is claimed to be caused by the negligence of structural inconsistency. In theory, scaling of pure acoustic 
modes is usually accurate; if the original cabin is downscaled by a specific numeric value, the original frequency of each acoustic mode will generally shift upwards by the multiple of this numeric value. This is similar in the context of a simple panel. However, in complex structures, it is not that simple because structural vibration fields are a combination of different elastic waves with some being dispersive and some being otherwise. Hence, it is difficult to establish a linear relationship.

Evidently, numerous analytical, numerical, and experimental considerations are crucial to arrive at the proposed QUASICAR model. This, in turn, makes the whole process laborious for the industry to even consider. Therefore, practicality of this approach in automobiles or armored vehicles is still far away since much time may be consumed to fine-tune the model.

\section{The Emergence of Acoustic Metamaterials}

Acoustic metamaterial (AM) is a recent research topic and is still in its infancy stage. Since the start of the current millennium year, there has been a rapid rise in research interest on AMs due to the exceptional phenomena exhibited for the first time in history. The mechanical properties of such metamaterials are basically governed by their carefully engineered physical structure rather than their chemical composition, which is the case in natural materials for the latter. Their construction is analogous to traditional honeycomb panels, which consists of periodic unit cells. In addition, each unit cell has a locally resonant structure, which exhibits exceptional phenomena at resonance; either negative effective bulk modulus or negative effective mass density. If properly designed, both characteristics can be achieved and the propagation of sound waves will be prohibited through the AM.

Dating back to 1960s, Veselago [1968] first demonstrated the preliminary concept analytically, where a negative refraction index is attainable if a medium exhibits both negative permeability and negative permittivity simultaneously. Unfortunately, his mathematical formulations remained as an abstract for over three decades before being realized experimentally by Pendry [2000]. In his work, he challenged the limitations of conventional lens by proposing a so-called nanoscale "superlens", which exhibits negative refraction index in the frequency bandwidth of visible light. At that moment, such phenomenon is only observed in the frequency bandwidth of microwave. During that period, most works focused on a frequency range that is in orders of magnitude higher than the audible frequency range $(20 \mathrm{~Hz}$ to $20 \mathrm{kHz})$ of humans. Liu et al. [2000] deviated from the mainstream and proposed an AM that attenuates noise in the mid frequency range $(400$ and $1100 \mathrm{~Hz})$ experimentally. The AM composes of periodically arranged high density spherical cores (lead) uniformly coated with a layer of soft and elastic material (silicone rubber). By specifying the size and geometry of each unit cell, noise attenuation at targeted frequencies is achieved. Their work was, however, a small-scale study in the laboratory. Their pioneering work brought forth a new field of research in various disciplines such as transformation optics [Jiang et al., 2009; Kinsler and McCall, 2015; Lu et al., 2009], 
energy harvesting [Chen et al., 2014], noise barriers [Garcia-Chocano et al., 2012; Garcia-Chocano and Sanchez-Dehesa, 2013; Ho et al., 2003; Lagarrigue et al., 2013; Sanchez-Dehesa et al., 2011] and vibration/shock mitigation [Akl and Baz, 2012; Baravelli and Ruzzene, 2013; Huang and Sun, 2010; Huang et al., 2009; Huang and Sun, 2009; Kim et al., 2015; Pai et al., 2014; Peng and Pai, 2014; Reynolds and Daley, 2014; Sharma and Sun, 2015; Tan et al., 2012].

The consideration of using AMs specifically for cavity noise control has been reported recently in two separate works. The first work proposed a one-dimensional AM, which constitutes of a five-mass array with each attached to a slender stem mounted onto the bottom surface of a small rectangular enclosure [Islam and Newaz, 2012]. The other work fabricated a cubic enclosure with its interior surfaces made up of periodically arranged locally resonant structures [Claeys et al., 2014]. Both successfully demonstrated the excellent acoustic attenuation of AM in the mid and high frequency range. Recent works moved to explore the possibility of low frequency noise. Two of such examples include the work of Naify et al. [2010, 2011a, 2011b, 2012, 2013] and Mei et al. [2012]. In general, their proposed AM constitutes of each unit cell being a thin membrane with a single or multiple platelets attached to its surface. This is better named as a membrane-platelet assembly. By tuning the mass of the platelet, noise attenuation can be achieved at targeted frequency below $200 \mathrm{~Hz}$. Yang et al. [2010] demonstrated noise attenuation at as low as $50 \mathrm{~Hz}$.

Till date, no existing natural materials are able to exhibit similar behavior at such small physical dimensions. This becomes advantageous when design requirements turn out to be so stringent in certain scenarios that no natural materials meet the necessary acoustical properties. From literature, it is evident that all these works remained as a laboratory study. As such, an extensive amount of exploratory works is definitely imperative to realize the full potential of AMs. Moreover, although positive results are observed from these reported studies, one should also include other vital factors in their design such as product reliability and ease of manufacturing. If the design is unreliable in practical applications or under certain environmental conditions, an increase in maintenance rate is likely to be inevitable. Clearly, this is undesirable for any potential investors. Additionally, the production of highly complex AMs may be challenging despite the availability of existing additive manufacturing techniques. Apart from this, the selection of the printing material must be cautiously considered as well since it affects the overall weight of the design. Generally speaking, designing an AM to meet industrial requirements - particularly for the defense platforms - may likely be a huge hurdle to overcome mainly due to the exposure to harsh environmental conditions and possible restriction of troops mobility within the vehicle.

\section{Conclusion}

From literature, it is evident that there are numerous approaches for tackling such problems; each having its own benefits and drawbacks. Psychoacoustics may be a 
good approach but is only feasible when the cabin noise is not excessively high such that safety exposure limit is exceeded. This is likely the case in armored vehicles since crew noise is usually on or near the borderline of safety exposure limit of $85 \mathrm{dBA}$. As such, higher priority should always be placed on crew noise reduction rather than perception studies and/or noise signature shaping.

Dimensional analysis can be a great tool in predicting cabin noise and structural/acoustic modes but when considered in detail, it is clearly time consuming and tedious to fine-tune the downscale model. Instead, these predictions can be performed more efficiently and effectively by numerical methods - namely FEM and BEM. Furthermore, the addition of PACA seems to be a highly reliable approach in identifying problematic panels that are contributing largely to the cabin noise. However, its functionality is only limited to source detection and hence further structural modifications or acoustical treatments are still necessary to improve cabin noise. These are generally done by passive or active solutions.

For active controls, their acoustical performance in the low frequency range $(<$ $200 \mathrm{~Hz}$ ) is evident as proven by numerous works. However, they are yet to be wellaccepted by the automotive and defense industry due to several challenges such as complex hardware configurations, high installation/implementation cost, nonintuitive software, complex algorithms, unique controller for different cabins, need for external power source, and nonruggedized control systems. As such, passive controls are still more reliable and stable in terms of acoustical performance. This is analogous to the preference of a fully-mechanical system as compared to a mechatronics system in the context of industrial product design. Active controls will be even more challenging to be implemented in armored vehicles as compared to commercial vehicles since they have to be sufficiently ruggedized to withstand harsh environmental conditions experienced commonly during mobility. Clearly, more time and research efforts are required to prove its practicality.

For passive controls, they are widely used in various industries, which is evident from their reliability and stability over active controls. However, they are not as effective in attenuating low frequency noise $(<200 \mathrm{~Hz})$. For example, a typical sound absorption material will require a very thick depth to at least reduce cabin noise noticeably at such long wavelength. As such, cabin space and vehicular weight are affected. Moreover, they deteriorate at a faster rate with time when exposed to harsh environmental conditions. This adversely affects their optimum acoustical performance and lead to higher maintenance cost, which is typically the case in armored vehicles. As also highlighted, other passive solutions face similar issues.

In light of the drawbacks of current industrial practices, the potential of AM is highlighted as a possible alternative for low frequency cabin noise control due to its exceptional phenomena. As highlighted, numerous works have demonstrated its acoustical performance in the lower frequency range without requiring a very thick specimen that is comparable to the wavelength of interest. The main challenge 
is the extension of existing works out of the laboratory to explore the feasibility of practical applications. As low frequency cabin noise is still a concern to date, preliminary works on AM may shed some light on its potential as an alternative solution to improve cabin noise in automobiles and armored vehicles; leading to better passenger comfort. Apart from merely achieving attractive results in the low frequency range $(<500 \mathrm{~Hz})$, the consideration of a few essential factors pertaining to manufacturability and reliability should also be considered for realization. Consequently, if these factors are considered in the design process of an AM, a potential approach for cabin noise control in automobiles and armored vehicles may emerge in the future.

\section{Acknowledgments}

The authors gratefully acknowledge the financial support provided by Singapore Economic Development Board (EDB) and Singapore Technologies Kinetics Ltd (ST Kinetics) under the EDB Industrial Postgraduate Program (EDB-IPP) (Grant No. COY-15-IPP/120005).

\section{References}

Akl, W. and Baz, A. M. [2012] "Analysis and experimental demonstration of an active acoustic metamaterial cell," Journal of Applied Physics 111(4), 044505.

Allman-Ward, M., Williams, R., Dunne, G. and Jennings, P. [2004] "The evaluation of vehicle sound quality using an NVH simulator," Proceedings of 33rd International Congress and Exposition on Noise Control Engineering, Prague, Czech Republic.

Arenas, J. P. and Crocker, M. J. [2010] "Recent trends in porous sound-absorbing materials," Sound and Vibration, 12-17.

Atalla, N. and Bernhard, R. J. [1994] "Review of numerical solutions for low-frequency structural-acoustic problems," Applied Acoustics 43(3), 271-294.

Atalla, N., Panneton, R., Sgard, F. C. and Olny, X. [2001] "Acoustic absorption of macroperforated porous materials," Journal of Sound and Vibration 243(4), 659-678.

Balachandran, B., Sampath, A. and Park, J. [1996] "Active control of interior noise in a three-dimensional enclosure," Smart Materials and Structures 5(1), 89-97.

Baravelli, E. and Ruzzene, M. [2013] "Internally resonating lattices for bandgap generation and low-frequency vibration control," Journal of Sound and Vibration 332(25), 65626579.

Blanchet, D. and Caillet, A. [2014] "Full frequency noise and vibration control onboard ships," Proceedings of 21st International Congress on Sound and Vibration, Beijing, China, pp. 1-8.

Boily, S. and Charron, F. [1999] "The vibroacoustic response of a cylindrical shell structure with viscoelastic and poroelastic materials," Applied Acoustics 58(2), 131-152.

Bravo, T., Maury, C. and Pinhede, C. [2012] "Vibroacoustic properties of thin microperforated panel absorbers," The Journal of the Acoustical Society of America 132(2), 789-798.

Bravo, T., Maury, C. and Pinhede, C. [2013] "Enhancing sound absorption and transmission through flexible multi-layer micro-perforated structures," The Journal of the Acoustical Society of America 134(5), 3663-3673. 
Bruneau, M., Garing, C. H. and Leblond, H. [1985] "Quality factor and boundary-layer attenuation of lower order modes in acoustic cavities," Journal de Physique 46(7), 1079-1085.

Bullmore, A. J., Nelson, P. A. and Elliott, S. J. [1990] "Theoretical studies of the active control of propeller-induced cabin noise," Journal of Sound and Vibration 140(2), 191-217.

Caillet, J., Marrot, F., Unia, Y. and Aubourg, P. A. [2012] "Comprehensive approach for noise reduction in helicopter cabins," Aerospace Science and Technology 23(1), 17-25.

Cao, J. [2005] "Numerical assessment of interior booming noise and noise reduction in an all-terrain tractor cabin," M.S. thesis, Industrial Systems Engineering Department, University of Regina, Saskatchewan, Canada.

Chandra, N., Raja, S. and Gopal, K. V. N. [2015] "A comprehensive analysis on the structural-acoustic aspects of various functionally graded plates," International Journal of Applied Mechanics 7(5), 1550072.

Cheer, J. and Elliott, S. J. [2013] "The design and performance of feedback controllers for the attenuation of road noise in vehicles," International Journal of Acoustics and Vibration 19(3), 155-164.

Chen, S. M., Wang, D. F. and Lei, Y. F. [2011] "Automotive interior noise prediction based on single sound cavity using statistical energy analysis method," Noise 6 Vibration Worldwide 42(11), 36-43.

Chen, S. M., Wang, D. F. and Zan, J. M. [2011] "Interior noise prediction of the automobile based on hybrid FE-SEA method," Mathematical Problems in Engineering 2011, 327170 .

Chen, Z. S., Guo, B., Yang, Y. M. and Cheng, C. C. [2014] "Metamaterials-based enhanced energy harvesting: A review," Physica B: Condensed Matter 438, 1-8.

Cherng, J. G., Yin, G., Bonhard, R. B. and French, M. [2002] "Characterization and validation of acoustic cavities of automotive vehicles," Proceedings of 2002 IMAC-XX: Conference $8 \mathcal{E}$ Exposition on Structural Dynamics, Los Angeles, California, pp. 290294.

Chintapalli, V. S. N. R. and Padmanabhan, C. [2014] "An experimental investigation of cavity noise control using mistuned Helmholtz resonators," Proceedings of 43rd International Congress on Noise Control Engineering, Melbourne, Australia.

Choi, H. G., Kang, S. W., Lee, S. H. and Lee, J. M. [1997] "Coupling analysis of interior noise of a vehicle with a roof-gap-trim effect," Proceedings of 1997 IMAC XV: 15th International Modal Analysis Conference, Orlando, Florida, pp. 444-449.

Citarella, R., Federico, L. and Cicatiello, A. [2007] "Modal acoustic transfer vector approach in a FEM-BEM vibro-acoustic analysis," Engineering Analysis with Boundary Elements 31(3), 248-258.

Claeys, C. C., Pluymers, B., Sas, P. and Desmet, W. [2014] "Design of a resonant metamaterial based acoustic enclosure," Proceedings of 26th International Conference on Noise and Vibration Engineering, Leuven, Belgium, pp. 3351-3358.

Cox, T. J. and D'Antonio, P. [2009] Acoustic Absorbers and Diffusers: Theory, Design and Application (Taylor \& Francis, New York).

Cummings, A. [1992] "The effects of a resonator array on the sound field in a cavity," Journal of Sound and Vibration 154(1), 25-44.

Demirkan, O. [2010] "The effects of structural modifications on acoustic characteristics of enclosed bodies," M.S. thesis, Mechanical Engineering Department, Middle East Technical University, Ankara, Turkey.

Department of Defense [2015] MIL-STD-1474E: Design Criteria Standard Noise Limits, USA. 
Desmet, W., Pluymers, B. and Sas, P. [2003] "Vibro-acoustic analysis procedures for the evaluation of the sound insulation characteristics of agricultural machinery cabins," Journal of Sound and Vibration 266(3), 407-441.

Dhandole, S. D., and Modak, S. V. [2007] "Review of vibro-acoustics analysis procedures for prediction of low frequency noise inside a cavity," Proceedings of 2007 IMAC-XXV: Conference $\&$ Exposition on Structural Dynamics, Orlando, Florida.

Doria, A. [1995] "Control of acoustic vibrations of an enclosure by means of multiple resonators," Journal of Sound and Vibration 181(4), 673-685.

Dorling, C. M., Eatwell, G. P., Hutchins, S. M., Ross, C. F. and Sutcliffe, S. G. C. [1989] "A demonstration of active noise reduction in an aircraft cabin," Journal of Sound and Vibration 128(2), 358-360.

Doutres, O., Atalla, N. and Osman, H. [2015] "Transfer matrix modeling and experimental validation of cellular porous material with resonant inclusions," The Journal of the Acoustical Society of America 137(6), 3502-3513.

Dowell, E. H., Gorman, G. F. and Smith, D. A. [1977] "Acoustoelasticity: General theory, acoustic natural modes and forced response to sinusoidal excitation, including comparisons with experiment," Journal of Sound and Vibration 52(4), 519-542.

Driesch, P. L. [2002] "Acoustic control in enclosures using optimally designed Helmholtz resonators," Ph.D. thesis, Mechanical and Nuclear Engineering Department, The Pennsylvania State University, State College, Pennsylvania.

Du, J. T., Li, W. L., Liu, Z. G., Xu, H. A. and Ji, Z. L. [2011] "Acoustic analysis of a rectangular cavity with general impedance boundary conditions," The Journal of the Acoustical Society of America 130(2), 807-817.

Egab, L., Wang, X. and Mohammad, F. [2014] "Acoustical characterisation of porous sound absorbing materials: A review," International Journal of Vehicle Noise and Vibration 10(1-2), 129-149.

Elliott, S. J. [2008] "A review of active noise and vibration control in road vehicles," Technical memorandum, Institute of Sound and Vibration Research, University of Southampton, Southampton, England.

Elliott, S. J., Nelson, P. A., Stothers, I. M. and Boucher, C. C. [1989] "Preliminary results of in-flight experiments on the active control of propeller-induced cabin noise," Journal of Sound and Vibration 128(2), 355-357.

Elliott, S. J., Nelson, P. A., Stothers, I. M. and Boucher, C. C. [1990] "In-flight experiments on the active control of propeller-induced cabin noise," Journal of Sound and Vibration 140(2), 219-238.

Esteve, S. J. and Johnson, M. E. [2002] "Reduction of sound transmission into a circular cylindrical shell using distributed vibration absorbers and Helmholtz resonators," The Journal of the Acoustical Society of America 112(6), 2840-2848.

Esteve, S. J. and Johnson, M. E. [2005] "Adaptive Helmholtz resonators and passive vibration absorbers for cylinder interior noise control," Journal of Sound and Vibration 288(4-5), 1105-1130.

European Automotive Research Partners Association [2013] "New NVH analysis tools and noise abatement technologies," Brussels, Belgium.

Everstine, G. C. [1997] "Finite element formulatons of structural acoustic problems," Computers \& Structures 65(3), 307-321.

Fahy, F. J. and Schofield, C. [1980] "Note on the interaction between a Helmholtz resonator and an acoustic mode of an enclosure," Journal of Sound and Vibration 72(3), 365-378.

Fenech, B., Keith, G. M. and Jacobsen, F. [2006] "The use of microperforated plates to attenuate cavity resonances," The Journal of the Acoustical Society of America 120(4), $1851-1858$. 
Forssen, J., Tober, S., Corakci, A. C., Frid, A. and Kropp, W. [2012] "Modelling the interior sound field of a railway vehicle using statistical energy analysis," Applied Acoustics 73(4), 307-311.

Franco, L. [1991] "Low frequency noise control in car cabin by means of the luggage compartment resonator," Applied Acoustics 32(1), 23-34.

Fuller, C. R. and Jones, J. D. [1987] "Experiments on reduction of propeller induced interior noise by active control of cylinder vibration," Journal of Sound and Vibration 112(2), 389-395.

Fuller, C. R., Maillard, J. P., Mercadal, M. and von Flotow, A. H. [2000] "Control of aircraft interior noise using globally detuned vibration absorbers," Journal of Sound and Vibration 203(5), 745-761.

Fuller, C. R., Snyder, S. D., Hansen, C. H. and Silcox, R. J. [1992] "Active control of interior noise in model aircraft fuselages using piezoceramic actuators," American Institute of Aeronautics and Astronautics Journal 30(11), 2613-2617.

Garcia-Chocano, V. M., Cabrera, S. and Sanchez-Dehesa, J. [2012] "Broadband sound absorption by lattices of microperforated cylindrical shells," Applied Physics Letters 101(2012), 1-5.

Garcia-Chocano, V. M. and Sanchez-Dehesa, J. [2013] "Optimum control of broadband noise by arrays of cylindrical units made of a recycled material," Applied Acoustics 74(1), 58-62.

Gentry, C. A., Guigou, C. and Fuller, C. R. [1997] "Smart foam for applications in passiveactive noise radiation control," The Journal of the Acoustical Society of America 101(4), 1771-1778.

Genuit, K. [2004] "The sound quality of vehicle interior noise: A challenge for the NVHengineers," International Journal of Vehicle Noise and Vibration 1(1/2), 158-168.

Georgiev, V. B., Krylov, V. V. and Winward, R. E. T. B. [2006a] "Simplified modelling of vehicle interior noise: Comparison of analytical, numerical and experimental approaches," Journal of Low Frequency Noise, Vibration and Active Control 25(2), 69-92.

Georgiev, V. B., Ranavaya, R. L. and Krylov, V. V. [2006b] "Experimental and numerical investigation of structure borne interior noise in a simplified vehicle model," Proceedings of the Institute of Acoustics 26(1), 412-421.

Gerner, C., Sachau, D., and Breitbach, H. [2003] "Active noise control in a semi-enclosure within an aircraft cabin," Proceedings of 10th International Congress on Sound and Vibration, Stockholm, Sweden, pp. 3721-3726.

Grewal, A., Zimcik, D. G., Hurtubise, L. and Leigh, B. [2000] "Active cabin noise and vibration control for turboprop aircraft using multiple piezoelectric actuators," Journal of Intelligent Materials Systems and Structures 11(6), 438-447.

Guigou, C. and Fuller, C. R. [1999] "Control of aircraft interior broadband noise with foam-PVDF smart skin," Journal of Sound and Vibration 220(3), 541-557.

Guo, R., Zhang, L. J., Zhao, J. and Zhou, H. [2012] "Interior structure-borne noise reduction by controlling the automotive body panel vibration," Proceedings of the Institution of Mechanical Engineers, Part D: Journal of Automobile Engineering 226(7), 943-956.

Halvorsen, W. G. and Emborg, U. [1989] "Interior noise control of the Saab 340 Aircraft," SAE Technical Paper, 891080.

Han, X., Guo, Y. J., Yu, H. D. and Zhu, P. [2012] "Interior sound field refinement of a passenger car using modified panel acoustic contribution analysis," International Journal of Automotive Technology 13(2), 293-300.

Hansen, C. H. [2005] "Current and future industrial applications of active noise control," Noise Control Engineering Journal 53(5), 181-198. 
Hatano, S. and Hashimoto, T. [2000] "Booming index as a measure for evaluating booming sensation," Proceedings of 29th International Congress and Exhibition on Noise Control Engineering, Nice, France, pp. 1-5.

Hirsch, S. M., Jayachandran, V. and Sun, J. Q. [1998] "Structural-acoustic control for quieter aircraft interior: Smart trim technology," Composite Structures 42, 189202.

Ho, K. M., Cheng, C. K., Yang, Z. Y., Zhang, X. X. and Sheng, P. [2003] "Broadband locally resonant sonic shields," Applied Physics Letters 83(26), 5566-5568.

Hoshino, H. [2000] "Balance of car interior noise components in consideration of masking effect," Proceedings of 29th International Congress and Exhibition on Noise Control Engineering, Nice, France, pp. 409-414.

Howard, C. Q., Hansen, C. H. and Zander, A. [2005] "Vibro-acoustic noise control treatments for payload bays of launch vehicles: Discrete to fuzzy solutions," Applied Acoustics 66(11), 1235-1261.

Hu, K., Wang, Y. S., Guo, H. and Chen, H. [2014] "Sound quality evaluation and optimization for interior noise of rail vehicle," Advances in Mechanical Engineering 2014(6), 820875 .

Huang, G. L. and Sun, C. T. [2010] "Band gaps in a multiresonator acoustic metamaterial," Journal of Vibration and Acoustics 132(3), 1-6.

Huang, H. H. and Sun, C. T. [2009] "Wave attenuation mechanism in an acoustic metamaterial with negative effective mass density," New Journal of Physics 11, 013003.

Huang, H. H., Sun, C. T. and Huang, G. L. [2009] "On the negative effective mass density in acoustic metamaterials," International Journal of Engineering Science 47(4), 610-617.

Huang, Y. M. and Chen, C. C. [2000] "Optimal design of dynamic absorbers on vibration and noise control of the fuselage," Computers 83 Structures 76, 691-702.

Huang, Y. M. and Fuller, C. R. [1997] "The effects of dynamic absorbers on the forced vibration of a cylindrical shell and its coupled interior sound field," Journal of Sound and Vibration 200(4), 401-418.

Huang, Y. M. and Fuller, C. R. [1998] "Vibration and noise control of the fuselage via dynamic absorbers," Journal of Vibration and Acoustics 120(2), 496-502.

Hughes, B. [2009] "What happened to active noise control?," Noise ES Vibration Worldwide $40(4), 2$.

Islam, M. T. and Newaz, G. [2012] "Metamaterial with mass-stem array in acoustic cavity," Applied Physics Letters 100(1), 011904.

Jahani, K., Beigmoradi, S., Ramezani, A. and Hajabdollahi, H. [2013] "Panel contribution analysis for a sedan car using numerical simulations," Proceedings of 20th International Congress on Sound 63 Vibration, Bangkok, Thailand.

Jiang, W. X., Yao, J. and Cui, T. J. [2009] "Anisotropic metamaterial devices," Materials Today 12(12), 26-33.

Kamci, G. [2009] "Vibroacoustic modeling of a commercial vehicle to reduce the interior noise level," M.S. thesis, Mechanical Engineering Department, Koc University, Istanbul, Turkey.

Kang, S. W., Lee, J. M. and Kim, S. H. [2000] "Structural-acoustic coupling analysis of the vehicle passenger compartment with the roof, air-gap, and trim boundary," Journal of Vibration and Acoustics 122, 196-202.

Kestell, C. D. [2000] "Active control of sound in a small single engine aircraft cabin with virtual error sensors," Ph.D. thesis, Mechanical Engineering Department, Adelaide University, Adelaide, Australia. 
Kim, E. H., Han, Y., Kim, N. and Yang, J. K. [2015] "Nonlinear stress wave propagation in 3D woodpile elastic metamaterials," International Journal of Solids and Structures $\mathbf{5 8}, 128-135$.

Kim, J. H., Lee, J. K., Im, B. S. and Cheong, C. C. [1999] "Modeling of piezoelectric smart structures including absorbing materials for cabin noise problems," Proceedings of SPIE 3667, 524-529.

Kim, S. H., Hong, S. Y., Song, J. H. and Joo, W. H. [2012] "Interior noise analysis of a construction equipment cabin based on airborne and structure-borne noise predictions," Journal of Mechanical Science and Technology 26(4), 1003-1009.

Kim, S. H. and Lee, J. M. [1998] "A practical method for noise reduction in a vehicle passenger compartment," Journal of Vibration and Acoustics 120, 199-205.

Kincaid, R. K., Laba, K. E. and Padula, S. L. [1997] "Quelling cabin noise in turboprop aircraft via active control," Journal of Combinatorial Optimization 1(3), 229-250.

Kinsler, P. and McCall, M. W. [2015] "The futures of transformations and metamaterials," Photonics and Nanostructures - Fundamentals and Applications 15, 10-23.

Kipp, C. R. and Bernhard, R. J. [1987] "Prediction of acoustical behavior in cavities using an indirect boundary element method," Journal of Vibration, Acoustics, Stress, and Reliability in Design 109(1), 22-28.

Kochan, K., Kletschkowski, T., Sachau, D. and Breitbach, H. [2010] "Active noise control in a semi-closed aircraft cabin," Noise \&6 Vibration Worldwide 41(3), 8-20.

Kochan, K., Sachau, D. and Breitbach, H. [2011] "Robust active noise control in the loadmaster area of a military transport aircraft," The Journal of the Acoustical Society of America 129(5), 3011-3019.

Kopuz, S. and Lalor, N. [1995] "Analysis of interior acoustic fields using the finite element method and the boundary element method," Applied Acoustics 45(3), 193210.

Krylov, V. V. [2002] "Simplified analytical models for prediction of vehicle interior noise," Proceedings of International Conference on Noise and Vibration Engineering 2002, Leuven, Belgium, pp. 1973-1980.

Krylov, V. V. [2004] "Simplified reduced-scale modelling of vehicle interior noise," Proceedings of 33rd International Congress and Exposition on Noise Control Engineering, Prague, Czech Republic.

Krylov, V. V., Walsh, S. J. and Winward, R. E. T. B. [2003] "Modelling of vehicle interior noise at reduced scale," Proceedings of Euronoise 2003, Naples, Italy.

Lagarrigue, C., Groby, J. P. and Tournat, V. [2013] "Sustainable sonic crystal made of resonating bamboo rods," The Journal of the Acoustical Society of America 133(1), $247-254$.

Lalor, N., and Priebsch, H. H. [2007] "The prediction of low- and mid-frequency internal road vehicle noise: A literature survey," Proceedings of the Institution of Mechanical Engineers, Part D: Journal of Automobile Engineering 221(3), 245-269.

Lane, S. A., Clark, R. L. and Southward, S. C. [2000] "Active control of low frequency modes in an aircraft fuselage using spatially weighted arrays," Journal of Vibration and Acoustics 122(3), 227-234.

Lane, S. A., Richard, R. E. and Kennedy, S. J. [2005] "Fairing noise control using tubeshaped resonators," Journal of Spacecraft and Rockets 42(4), 640-646.

Lanoye, R., Vermeir, G., Lauriks, W., Sgard, F. C. and Desmet, W. [2008] "Prediction of the sound field above a patchwork of absorbing materials," The Journal of the Acoustical Society of America 123(2), 793-802. 
Lee, H. H. and Lee, S. K. [2009] "Objective evaluation of interior noise booming in a passenger car based on sound metrics and artificial neural networks," Applied Ergonomics 40(5), 860-869.

Lee, S. K., Kim, T. G. and Lee, U. [2006] "Sound quality evaluation based on artificial neural network," in Advances in Natural Computation (Springer, Berlin), pp. 545-554.

Lee, S. K., Lee, S. M., Kang, I. D. and Shin, T. J. [2014] "Active sound design for a passenger car based on adaptive order filter," Proceedings of 43rd International Congress on Noise Control Engineering, Melbourne, Australia.

Li, D. and Cheng, L. [2007] "Acoustically coupled model of an enclosure and a Helmholtz resonator array," Journal of Sound and Vibration 305(1-2), 272-288.

Li, D., Cheng, L., Yu, G. H. and Vipperman, J. S. [2007] "Noise control in enclosures: Modeling and experiments with T-shaped acoustic resonators," The Journal of the Acoustical Society of America 122(5), 2615-2625.

Li, D., Zhang, X. H., Cheng, L. and Yu, G. H. [2010] "Effectiveness of T-shaped acoustic resonators in low-frequency sound transmission control of a finite double-panel partition," Journal of Sound and Vibration 329(22), 4740-4755.

Liu, J. W. and Herrin, D. W. [2010] "Enhancing micro-perforated panel attenuation by partitioning the adjoining cavity," Applied Acoustics 71(2), 120-127.

Liu, N. N., Wang, Y. S. and Shi, L. [2013] "Annoyance evaluation of subway interior noise based on psychoacoustical indices," Noise $\&$ Vibration Worldwide 44(9), 18-24.

Liu, Z. S., Lee, H. P. and Lu, C. [2006] "Passive and active interior noise control of box structures using the structural intensity method," Applied Acoustics 67(2), 112-134.

Liu, Z. S., Lu, C., Wang, Y. Y., Lee, H. P., Koh, Y. K. and Lee, K. S. [2006] "Prediction of noise inside tracked vehicles," Applied Acoustics 67(1), 74-91.

Liu, Z. Y., Zhang, X. X., Mao, Y. W., Zhu, Y. Y., Yang, Z. Y., Chan, C. T. and Sheng, P. [2000] "Locally resonant sonic materials," Science 289(5485), 1734-1736.

Lu, M. H., Feng, L. and Chen, Y. F. [2009] "Phononic crystals and acoustic metamaterials," Materials Today 12(12), 34-42.

Maa, D. Y. [2015] "Potential of microperforated panel absorber," The Journal of the Acoustical Society of America 104(5), 2861-2866.

MacMartin, D. G. [1996] "Collocated structural control for reduction of aircraft cabin noise," Journal of Sound and Vibration 190(1), 105-119.

Marburg, S. [2002] "Six boundary elements per wavelength: Is that enough?" Journal of Computational Acoustics 10(1), 25-51.

Martin, V. and Bodrero, A. [1997] "An introduction to the control of sound fields by optimising impedance locations on the wall of an acoustic cavity," Journal of Sound and Vibration 204(2), 331-357.

Martin, V., Vignassa, P., and Peseux, B. [1994] "Numerical vibro-acoustic modelling of aircraft for the active acoustic control of interior noise," Journal of Sound and Vibration 176(3), 307-332.

Marzbanrad, J. and Beyg, M. A. [2012] "Vehicle cabin noise simulation due to highfrequencies stimulation," International Journal of Automotive Engineering 2(2), $117-123$.

Mathur, G. P., and Troi, B. N. [1993] "Aircraft cabin noise reduction tests using active structural acoustic control," Proceedings of 15th Aeroacoustics Conference, Long Beach, California.

Mei, J., Ma, G. C., Yang, M., Yang, Z. Y., Wen, W. J. and Sheng, P. [2012] "Dark acoustic metamaterials as super absorbers for low-frequency sound," Nature Communications 3, 756 . 
Misol, M., Algermissen, S. and Monner, H. P. [2012] "Experimental investigation of different active noise control concepts applied to a passenger car equipped with an active windshield," Journal of Sound and Vibration 331(10), 2209-2219.

Missaoui, J. and Cheng, L. [1997] "A combined integro-modal approach for predicting acoustic properties of irregular-shaped cavities," Journal of the Acoustical Society of America 101(6), 3313-3321.

Missaoui, J. and Cheng, L. [1999] "Vibroacoustic analysis of a finite cylindrical shell with internal floor partition," Journal of Sound and Vibration 226(1), 101-123.

Muller, G. and Moser, M. [2013] Handbook of Engineering Acoustics (Springer, Berlin).

Naify, C. J. [2013] "Noise control using lightweight acoustic metamaterials," Proceedings of Meetings on Acoustics 19, 065017.

Naify, C. J., Chang, C. M., McKnight, G. and Nutt, S. R. [2010] "Transmission loss and dynamic response of membrane-type locally resonant acoustic metamaterials," Journal of Applied Physics 108(11), 114905.

Naify, C. J., Chang, C. M., McKnight, G. and Nutt, S. R. [2011a] "Transmission loss of membrane-type acoustic metamaterials with coaxial ring masses," Journal of Applied Physics 110(12), 124903.

Naify, C. J., Chang, C. M., McKnight, G. and Nutt, S. R. [2012] "Scaling of membrane-type locally resonant acoustic metamaterial arrays," The Journal of the Acoustical Society of America 132(4), 2784.

Naify, C. J., Chang, C. M., McKnight, G., Scheulen, F. and Nutt, S. R. [2011b] "Membranetype metamaterials: Transmission loss of multi-celled arrays," Journal of Applied Physics 109(10), 104902.

Nefske, J., Wolf, J. A. and Howell, L. J. [1982] "Structural-acoustic finite element analysis of passenger compartment: A review of current practice," Journal of Sound and Vibration 80(2), 247-266.

Niezrecki, C. and Cudney, H. H. [2001] "Feasibility to control launch vehicle internal acoustics using piezoelectric actuators," Journal of Intelligent Materials Systems and Structures 12(9), 647-660.

Noise \& Vibration Worldwide. [2002] "Applications of active noise and vibration control applications control in vehicles," Noise 85 Vibration Worldwide 33(8), 11-15.

Nopiah, Z. M., Junoh, A. K. and Ariffin, A. K. [2014] "Computational method for predicting the effects of vibrations to acoustical comfort in vehicle cabin," International Journal of Vehicle Noise and Vibration 10(1-2), 150-170.

Nopiah, Z. M., Junoh, A. K. and Ariffin, A. K. [2015] "Vehicle interior noise and vibration level assessment through the data clustering and hybrid classification model," Applied Acoustics 87, 9-22.

Nor, M. J. M., Fouladi, M. H., Nahvi, H. and Ariffin, A. K. [2008] "Index for vehicle acoustical comfort inside a passenger car," Applied Acoustics 69(4), 343-353.

Oh, S. H., Kim, H. S. and Park, Y. J. [2002] "Active control of road booming noise in automotive interiors," The Journal of the Acoustical Society of America 111(1), 180188.

Olatunbosun, O. A., Gauchia, A., Boada, M. J. L. and Diaz, V. [2011] "Dynamic performance analysis of a light van body-in-white structure," Proceedings of the Institution of Mechanical Engineers, Part D: Journal of Automobile Engineering 225(2), 167-177.

Pai, P. F., Peng, H. and Jiang, S. Y. [2014] "Acoustic metamaterial beams based on multi-frequency vibration absorbers," International Journal of Mechanical Sciences 79, 195-205. 
Palumbo, D., Cabell, R., Cline, J. and Sullivan, B. [2000] "Active structural acoustic control of interior noise on a Raytheon 1900D," Technical memorandum, Langley Research Center, NASA, Hampton, Virginia.

Pan, J. [1991] "Active control of noise transmission through a panel into a cavity. III: Effect of the actuator location," The Journal of the Acoustical Society of America 90(3), 1493-1501.

Pan, J., Elliott, S. J. and Baek, K. H. [1999] "Analysis of low frequency acoustic response in a damped rectangular enclosure," Journal of Sound and Vibration 223(4), 543-566.

Pan, J. and Hansen, C. H. [1991] "Active control of noise transmission through a panel into a cavity. II: Experimental study," The Journal of the Acoustical Society of America 90(3), 1488-1492.

Pan, J., Hansen, C. H. and Bies, D. A. [1990] "Active control of noise transmission through a panel into a cavity. I: Analytical study," The Journal of the Acoustical Society of America 87(5), 2098-2108.

Pang, F. Z., Song, H. B. and Miao, X. H. [2014] "Research on vibration and sound radiation characteristics of ship stiffened composite plate structure," Proceedings of 43rd International Congress on Noise Control Engineering, Melbourne, Australia, pp. 1-9.

Park, I. S. and Sohn, C. H. [2010] "Nonlinear acoustic damping induced by a half-wave resonator in an acoustic chamber," Aerospace Science and Technology 14(6), 442-450.

Park, J. H. and Sohn, C. H. [2009] "On optimal design of half-wave resonators for acoustic damping in an enclosure," Journal of Sound and Vibration 319(3-5), 807-821.

Park, S. H. [2013a] "A design method of micro-perforated panel absorber at high sound pressure environment in launcher fairings," Journal of Sound and Vibration 332(3), 521-535.

Park, S. H. [2013b] "Acoustic properties of micro-perforated panel absorbers backed by Helmholtz resonators for the improvement of low-frequency sound absorption," Journal of Sound and Vibration 332(20), 4895-4911.

Park, S. Y., Kim, S. H. and Kang, Y. J. [2010] "The effect of a local stiffener in the structural-acoustic coupled system," Proceedings of the Institution of Mechanical Engineers, Part C: Journal of Mechanical Engineering Science 224(C9), 1915-1931.

Paulraj, M. P., Yaacob, S., Andrew, A. M. and Othman, S. M. [2010] "Sound quality evaluation for vehicle interior noise based on psychoacoustic model," Proceedings of Challenges and Innovations in Information Technology, Erode, India, pp. 553-560.

Pendry, J. B. [2000] "Negative refraction makes a perfect lens," Physical Review Letters 85(18), 3966-3969.

Peng, H. and Pai, P. F. [2014] "Acoustic metamaterial plates for elastic wave absorption and structural vibration suppression," International Journal of Mechanical Sciences 89, 350-361.

Petyt, M. and Koopmann, G. H. [1977] "The acoustic modes of a rectangular cavity containing a rigid, incomplete partition," Journal of Sound and Vibration 53(1), 71-82.

Poh, S., Baz, A. M. and Balachandran, B. [1996] "Experimental adaptive control of sound radiation from a panel into an acoustic cavity using active constrained layer damping," Smart Materials \& Structures 5(5), 649-659.

Putra, A., Munawir, A. and Farid, W. M. [2015] "Corrected statistical energy analysis model for car interior noise," Advances in Mechanical Engineering 7(1), 304283.

Radic, N., Novak, C. and Ule, H. [2011] "Experimental evaluation of vehicle cabin noise using subjective and objective psychoacoustic analysis techniques," Journal of the Canadian Acoustical Association 39(4), 27-36.

Rao, M. D. [2003] "Recent applications of viscoelastic damping for noise control in automobiles and commercial airplanes," Journal of Sound and Vibration 262(3), 457-474. 
Reynolds, M. and Daley, S. [2014] "An active viscoelastic metamaterial for isolation applications," Smart Materials and Structures 23(4), 045030.

Richards, T. L. and Jha, S. K. [1979] "A simplified finite element method for studying acoustic characteristics inside a car cavity," Journal of Sound and Vibration 63(1), $61-72$.

Sacarcelik, O. [2004] "Acoustic devices for the active \& passive control of sound in a payload compartment," M.S. thesis, Mechanical Engineering Department, Virginia Polytechnic Institute and State University, Blacksburg, Virginia.

Sanchez-Dehesa, J., Garcia-Chocano, V. M., Torrent, D., Cervera, F., Cabrera, S. and Simon, F. [2011] "Noise control by sonic crystal barriers made of recycled materials," The Journal of the Acoustical Society of America 129(3), 1173-1183.

Sarigul, A. S. and Kiral, Z. [1999] "Interior acoustics of a truck cabin with hard and impedance surfaces," Engineering Analysis with Boundary Elements 23(9), 769-775.

Sas, P. and Dehandschutter, W. [2009] "Active structural and acoustic control of structureborne road noise in a passenger car," Noise ES Vibration Worldwide 30(5), 17-27.

Sestieri, A. and Carcaterra, A. [2013] "Vibroacoustic: The challenges of a mission impossible?," Mechanical Systems and Signal Processing 34(1-2), 1-18.

Sestieri, A., Vescovo, D. Del and Lucibello, P. [1984] "Structural-acoustic coupling in complex shaped cavities," Journal of Sound and Vibration 96(2), 219-233.

Sgard, F. C., Olny, X., Atalla, N. and Castel, F. [2005] "On the use of perforations to improve the sound absorption of porous materials," Applied Acoustics 66(6), 625-651.

Sharma, B. and Sun, C. T. [2015] "Impact load mitigation in sandwich beams using local resonators," Journal of Sandwich Structures and Materials, 18(1), 50-64.

Shende, V. [2014] "Analysis of research in consumer behavior of automobile passenger car customer," International Journal of Scientific and Research Publications 4(2), 1-8.

Shin, S. H., Ih, J. G., Hashimoto, T. and Hatano, S. [2009] "Sound quality evaluation of the booming sensation for passenger cars," Applied Acoustics 70(2), 309-320.

Shuku, T. and Ishihara, K. [1973] "The analysis of the acoustic field in irregularly shaped rooms by the finite element method," Journal of Sound and Vibration 29(1), 67-76.

Silcox, R. J., Lester, H. C. and Abler, S. B. [1989] "An evaluation of active noise control in a cylindrical shell," Journal of Vibration, Acoustics, Stress, and Reliability in Design 111(3), 337-342.

Simpson, M. A., Luong, T. M., Fuller, C. R. and Jones, J. D. [1991] "Full-scale demonstration tests of cabin noise reduction using active vibration control," Journal of Aircraft 28(3), 208-215.

Sohn, C. H. and Park, J. H. [2011] "A comparative study on acoustic damping induced by half-wave, quarter-wave, and Helmholtz resonators," Aerospace Science and Technology 15(8), 606-614.

Song, C. K., Hwang, J. K., Lee, J. M. and Hedrick, J. K. [2003] "Active vibration control for structural-acoustic coupling system of a 3-D vehicle cabin model," Journal of Sound and Vibration 267(4), 851-865.

Stanef, D. A., Hansen, C. H. and Morgans, R. C. [2004] "Active control analysis of mining vehicle cabin noise using finite element modelling," Journal of Sound and Vibration 277(1-2), 277-297.

Subramanian, S., Surampudi, R., Thomson, K. R. and Vallurupalli, S. [2004] "Optimization of damping treatment for structure borne noise reduction," Sound and Vibration, $14-17$.

Succi, G. P. [1987] "The interior acoustic field of an automobile cabin," Journal of the Acoustical Society of America 81(6), 1688-1694. 
Sun, J. Q., Norris, M. A., Rossetti, D. J. and Highfill, J. H. [1996] "Distributed piezoelectric actuators for shell interior noise control," Journal of Vibration and Acoustics 118(1), 676-681.

Sung, M. H., Lee, J. M., Kim, S. H., Park, D. C., Chung, S. G. and Kim, J. H. [1994] "A study on the vibration and interior noise characteristics of a passenger car," Proceedings of 1994 IMAC-XII: 12th International Modal Analysis Conference, Honolulu, Hawaii, pp. $1435-1440$.

Sutton, T. J., Elliott, S. J., McDonald, A. M. and Saunders, T. J. [1994] "Active control of road noise inside vehicles," Noise Control Engineering Journal 42(4), 137-147.

Suzuki, S., Maruyama, S. and Ido, H. [1989] "Boundary element analysis of cavity noise problems with complicated boundary conditions," Journal of Sound and Vibration 130(1), 79-91.

Tan, K. T., Huang, H. H. and Sun, C. T. [2012] "Optimizing the band gap of effective mass negativity in acoustic metamaterials," Applied Physics Letters 101(24), 241902.

Tichy, J. [1991] "Current and future issues of active noise control," The Acoustical Society of Japan 12(6), 255-262.

Toyoda, M., Kobatake, S. and Sakagami, K. [2015] "Numerical analyses of the sound absorption of cylindrical microperforated panel space absorbers with cores," The Journal of the Acoustical Society of America 138(6), 3531-3538.

Veselago, V. G. [1968] "The electrodynamics of substances with simultaneously negative values of permittivity and permeability," Soviet Physics Uspekhi 10(4), 509-514.

Vige, D. [2010] "Cabin sound package design and development," in Vehicle Noise and Vibration Refinement (Woodhead Publishing Limited, Cambridge, UK), pp. 286-317.

Wang, C. Q., Cheng, L., Pan, J. and Yu, G. H. [2010] "Sound absorption of a microperforated panel backed by an irregular-shaped cavity," The Journal of the Acoustical Society of America 127(1), 238-246.

Wang, Y. S., Lee, C. M., Kim, D. G. and Xu, Y. [2007] "Sound-quality prediction for nonstationary vehicle interior noise based on wavelet pre-processing neural network model," Journal of Sound and Vibration 299(4-5), 933-947.

Wang, Y. S., Liu, X. T. and Li, Y. [2010] "A vibro-acoustic coupling analysis of bus passenger compartment based on the finite element method," Proceedings of 2010 International Conference on Intelligent Computation Technology and Automation, Changsha, China, pp. 386-389.

Willemsen, A. M., Poradek, F. and Rao, M. D. [2009] "Reduction of noise in an excavator cabin using order tracking and ultrasonic leak detection," Noise Control Engineering Journal 57(5), 400-412.

Wu, J. H., Chen, H. L. and An, W. B. [2000] "A method to predict sound radiation from a plate-ended cylindrical shell excited by an external force," Journal of Sound and Vibration 237(5), 793-803.

Xu, M., Zhang, J. H., Lin, J. W., Guo, P., He, W. J. and Li, Z. W. [2015] "Prediction and control of the structure-borne noise of a vehicle based on an optimization technique of the vehicle-body model," Proceedings of the Institution of Mechanical Engineers, Part D: Journal of Automobile Engineering 229(11), 1457-1468.

Yang, C., Cheng, L. and Hu, Z. Y. [2015] "Reducing interior noise in a cylinder using micro-perforated panels," Applied Acoustics 95(1), 50-56.

Yang, Z. Y., Dai, H. M., Chan, N. H., Ma, G. C. and Sheng, P. [2010] "Acoustic metamaterial panels for sound attenuation in the 50-1000 Hz regime," Applied Physics Letters 96(4), 041906.

Yildirim, S. and Eski, I. [2008] "Sound quality analysis of cars using hybrid neural networks," Simulation Modelling Practice and Theory 16(4), 410-418. 
Yu, G. H. and Cheng, L. [2009] "Location optimization of a long T-shaped acoustic resonator array in noise control of enclosures," Journal of Sound and Vibration 328(1-2), $42-56$.

Yu, G. H., Li, D. and Cheng, L. [2008] "Effect of internal resistance of a Helmholtz resonator on acoustic energy reduction in enclosures," The Journal of the Acoustical Society of America 124(6), 3534-3543.

Yu, X., Cheng, L. and Guyader, J. L. [2014] "Modeling vibroacoustic systems involving cascade open cavities and micro-perforated panels," The Journal of the Acoustical Society of America 136(2), 659-670.

Yuksel, E., Kamci, G. and Basdogan, I. [2012] "Vibro-acoustic design optimization study to improve the sound pressure level inside the passenger cabin," Journal of Vibration and Acoustics 134(6), 061017.

Zhang, S. and Cheng, L. [2015] "Shape optimization of acoustic enclosures based on a Wavelet-Galerkin formulation," International Journal of Applied Mechanics 7(1), 1550009 .

Zhao, D., Ang, L. and Ji, C. Z. [2015] "Numerical and experimental investigation of the acoustic damping effect of single-layer perforated liners with joint bias-grazing flow," Journal of Sound and Vibration 342, 152-167.

Zhao, Y. J., Deng, X., Liu, S. Q., Shuai, R., Sun, Z. J., Guo, J. Q. and Xu, Y. [2013] "Interior noise prediction of high-speed train based on hybrid FE-SEA method," Proceedings of 11th International Workshop on Railway Noise, Uddevalla, Sweden, pp. 699-705.

Zheng, H. and Wei, Z. P. [2013] "Vibroacoustic analysis of stiffened plates with nonuniform boundary conditions," International Journal of Applied Mechanics 5(4), 1350046. 